\title{
A New 2-D Model of a Thin Annular Disk Using a Modified Assumption
}

\author{
Yichen Qian, Nick R. Harris, Peter Glynne-Jones, and Steve P. Beeby
}

\begin{abstract}
The work describes an improved 2-D model for a thin annulus by using a modified assumption with regard to coupled vibration. With this approach, the impedance spectrum and displacements due to radial modes, both in radial and thickness direction of a thin ring, are obtained. Bending displacement is investigated by finite element analysis (FEA) and matches our model. The bending in the thickness direction is coupled to radial modes and shows several node circles in the high radial overtone frequency range. The model is validated by FEA with excellent agreement between the new theory and FEA results.
\end{abstract}

\section{INTRODUCTION}

$\mathrm{P}$ IEZOELECTRIC ring transducers are widely used in the fields of underwater and medical applications [1]. Radial ( $\mathrm{R}$ ) only modes for thin ring structures have been well developed in the past decades and illustrated in previous literature [1]-[3]. Whereas, in these previous models, the $\mathrm{R}$ modes included no out-of-plane vibration (apart from Poisson effects), the more accurate model presented in this paper does and discovers that the modeled $\mathrm{R}$ modes effectively have a certain amount of bending in their mode shape, which is caused by the $\mathrm{R}$ mode and enhanced by the increase of the order of the $\mathrm{R}$ mode.

In our analytical model, the technique is based on a new assumption made from the kinematic relationship. The assumption presented here considers the coupled vibration in both thickness and radial direction; previous work [4]-[7] has used relationships that decouple the vibrations in thickness and radial direction and thus leads to inaccurate results. By finite element analysis (FEA) simulation (using ANSYS 11.0 software, ANSYS Inc., Canonsburg, PA), which has been shown to produce results close to experimental data [8], the analytical results from our model show a very good agreement with FEA results, with the coupled bending vibration obtained accurately in the thickness direction.

In addition, this bending is believed to be the main reason for the nonuniform thickness extensional (TE) vibration that is excited in the far frequency range that is often associated with high overtones of the $\mathrm{R}$ mode; the enhanced bending due to high-order $\mathrm{R}$ modes is thus strongly coupled into TE vibration. The assumption al-

Manuscript received December 18, 2008; accepted October 28, 2009.

Y. Qian, N. R. Harris, and S. P. Beeby are with the School of Electronics and Computer Science, University of Southampton, Southampton, UK (e-mail: kiwi.q@hotmail.com).

P. Glynne-Jones is with the School of Engineering Science, University of Southampton, Southampton, UK.

Digital Object Identifier 10.1109/TUFFC.2010.1422 lowing coupled vibration still may be valid for the TE mode and could serve as a step to obtain accurate results for the coupled TE vibration, but this has yet to be demonstrated.

\section{Model of Thin Ring Using A Modified ASSUMPTION}

Fig. 1 shows the structure of a ring defined within axissymmetric cylindrical coordinates. The angular displacement in $\theta$ direction (cylindrical coordinates) can be ignored due to symmetry [1]. The governing equations for piezoelectricity in cylindrical coordinates are given below:

$$
\begin{gathered}
T_{r}=c_{11}^{E} S_{r}+c_{12}^{E} S_{\theta}+c_{13}^{E} S_{z}+e_{31} \partial \varphi / \partial z \\
T_{\theta}=c_{12}^{E} S_{r}+c_{11}^{E} S_{\theta}+c_{13}^{E} S_{z}+e_{31} \partial \varphi / \partial z \\
T_{z}=c_{13}^{E}\left(S_{r}+S_{\theta}\right)+c_{33}^{E} S_{z}+e_{33} \partial \varphi / \partial z, \\
T_{r z}=c_{44}^{E} S_{r z}+e_{15} \partial \varphi / \partial r \\
D_{r}=e_{15} S_{r z}-\varepsilon_{11}^{S} \partial \varphi / \partial r \\
D_{z}=e_{31}\left(S_{r}+S_{\theta}\right)+e_{33} S_{z}-\varepsilon_{33}^{S} \partial \varphi / \partial z, \\
S_{r}=\partial u_{r} / \partial r, \quad S_{z}=\partial u_{z} / \partial z, \quad S_{\theta}=u_{r} / r, \\
S_{r z}=\partial u_{r} / \partial z+\partial u_{z} / \partial r \\
E_{r}=-\partial \varphi / \partial r, \quad E_{z}=-\partial \varphi / \partial z \\
\frac{\partial D_{r}}{\partial r}+\frac{1}{r} D_{r}+\frac{\partial D_{z}}{\partial z}=0,
\end{gathered}
$$

where $T_{i, j}$ is the stress in the defined direction, $S_{i, j}$ is the strain, $\varphi$ represents the electrical potential, $E$ is the electrical field, and $D$ is electric displacement; $c_{11}{ }^{E}, c_{12}{ }^{E}, \ldots$ $c_{44}{ }^{E}$ are the elastic stiffness constant; $e_{31}, e_{33}, e_{15}$ are the dielectric constants, $\varepsilon_{11} S$ and $\varepsilon_{33} S$ are the permittivity, $u_{r}$ and $u_{z}$ are the radial and thickness displacements, respectively. The differential equations for the 2-D problem are also given:

$$
\begin{aligned}
\frac{\partial T_{r}}{\partial r}+\frac{T_{r}-T_{\theta}}{r}+\frac{\partial T_{r z}}{\partial z} & =\rho \frac{\partial^{2} u_{r}}{\partial t^{2}} \\
\frac{\partial T_{r z}}{\partial r}+\frac{T_{r z}}{r}+\frac{\partial T_{z}}{\partial z} & =\rho \frac{\partial^{2} u_{z}}{\partial t^{2}} .
\end{aligned}
$$

Because the polarization is in the thickness direction and the ring is supposed to be thin, the classic Kirchhoff's theory is used, and several assumptions are made and summarized as follows [1], [9]: 


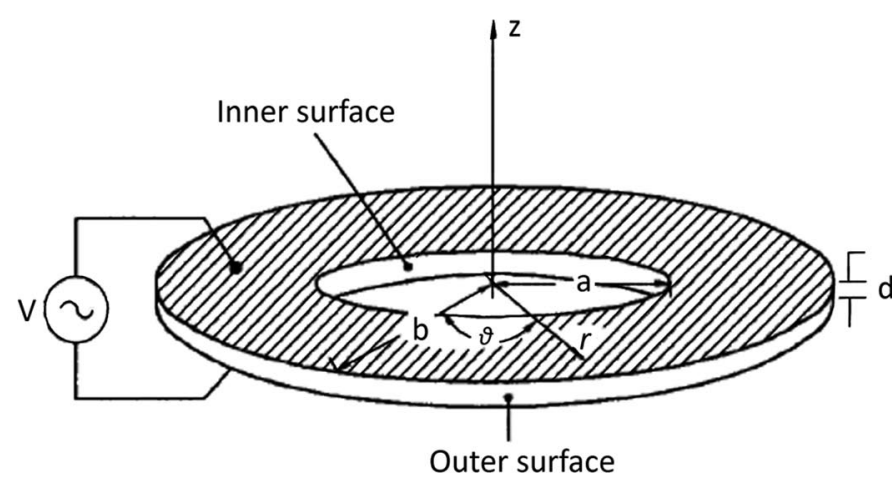

Fig. 1. The geometric structure of a thin ring.

$$
T_{z}=T_{r z}=E_{r}=D_{r}=0, \quad \varphi=V z / d, \quad S_{r z}=0 .(3)
$$

These assumptions are reasonable because the top and bottom surfaces of the thin ring hold the stress freeboundary condition. Because the thickness is also small, the stress quantities cannot depart much from zero. Likewise, the linear approximation to voltage through the thickness is also a result of the thin nature of the disk. The zero shear deformation $S_{r z}$ can be deduced by (1d). By the zero shear deformation, the relationship of both radial and thickness displacements can be obtained by classic kinematic relation [10], as expressed in following equation:

$$
u_{z}=\frac{\partial v(z)}{\partial z} \cdot w(r), \quad u_{r}=-v(z) \cdot \frac{\partial w(r)}{\partial r}
$$

where $v$ and $w$ are the functions dependent on $z$ and $r$, respectively. For the conventional assumption used in [4]-[7], the displacements $u_{r}$ and $u_{z}$ are decoupled and assumed to be functions only of radius $r$ and thickness $z$, respectively. The modified assumption made in (4) realizes the coupled vibration; both $r$ and $z$ determine the displacements. The new, coupled assumption is expected to offer more accurate results. FEA study is used here to verify the analytical model. The FEA representation is modeled in ANSYS and essentially 2-D, consisting of a rectangular element using Solid PLANE13 denoting the piezoelectric resonator, and with an axis-symmetric boundary at the circle center to represent the 3-D annular structure. A $1-\mathrm{V}$ harmonic voltage is applied onto the top surface of the resonator, and the bottom surface is grounded. Thus harmonic analyses can be applied to obtain impedance and displacement responses. The parameters of the FEA model are listed in Table I.

The FEA results show that the displacement $u_{z}$ in the thickness direction at fixed radius appears as linear function. This is because, although the component $\partial v(z) / \partial z$

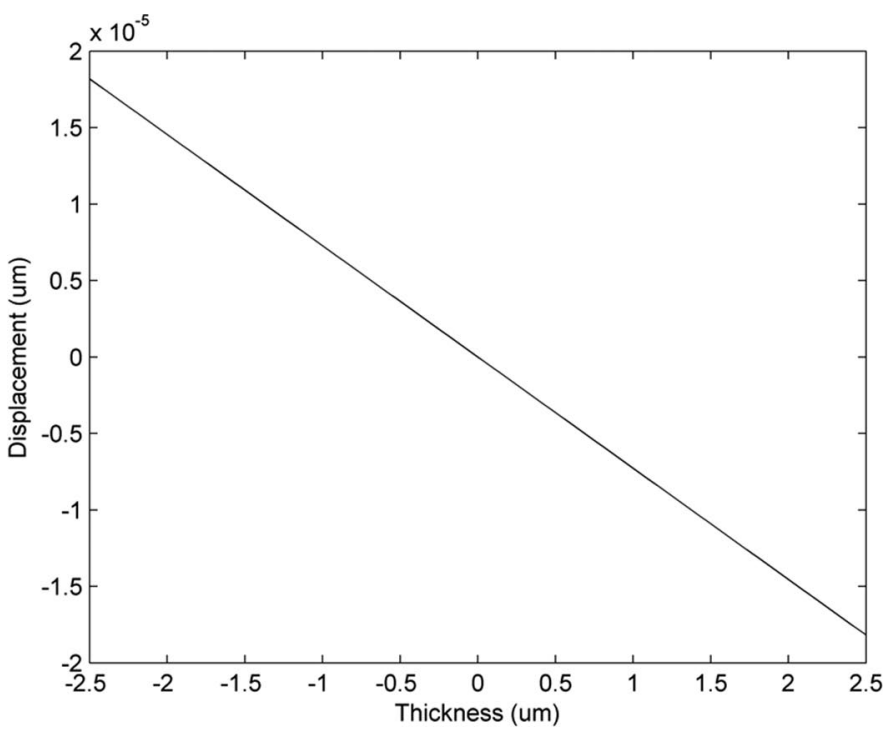

Fig. 2. $u_{z}$ at fixed radius $r$.

of $u_{z}$ may take the form of a sinusoidal function for thin rings, thickness $z$ is quite small compared with the radial wavelength, and this small range of $z$ allows a linear approximation.

As verified in Fig. 2, $u_{z}$ for fixed radius $r$ and fixed applied voltage $V$ is linear, which means $u_{z}$ can be expressed as

$$
u_{z}=\frac{\partial v(z)}{\partial z} \cdot w(r)=-z \cdot w(r)
$$

where the linear coefficient of $z$ is absorbed into $w(r)$ because this is constant at fixed $r$. Thus, by the deduction from (4), the radial displacement is obtained,

$$
u_{r}=-v(z) \cdot \frac{\partial w(r)}{\partial r}=\left(\frac{z^{2}}{2}+C\right) \frac{\partial w}{\partial r}
$$

where $C$ is an unknown constant generated from the integration. The elastic governing equations from (1a) to (1f) can be simplified through Kirchhoff's theory listed in (3). The simplified governing equations are obtained,

$$
\begin{gathered}
T_{r}=c_{11}^{p} \partial u_{r} / \partial r+c_{12}^{p} u_{r} / r+e_{31}^{p} \partial \varphi / \partial z \\
T_{r}=c_{12}^{p} \partial u_{r} / \partial r+c_{11}^{p} u_{r} / r+e_{31}^{p} \partial \varphi / \partial z \\
D_{z}=e_{31}^{p}\left(\partial u_{\mathrm{r}} / \partial r+u_{r} / r\right)-\varepsilon_{33}^{p} \partial \varphi / \partial z
\end{gathered}
$$

where the parameters with superscript $p$ are given as follows:

$$
\begin{aligned}
& c_{11}^{p}=c_{11}^{E}-c_{13}^{E}{ }^{2} \mathrm{e} c_{33}^{E}, \quad c_{12}^{p}=c_{12}^{E}-c_{13}^{E} / c_{33}^{E}, \\
& \varepsilon_{33}^{p}=\varepsilon_{33}^{S}-e_{33}^{2} / c_{33}^{E} \quad e_{31}^{p}=e_{31}-e_{33} c_{13}^{E} / c_{33}^{E} .
\end{aligned}
$$

TABLE I. Ring Transducer Parameters.

\begin{tabular}{lllll}
\hline PZT ceramic & Inner radius $(a)$ & Outer radius $(b)$ & Width $(w=b-a)$ & Thickness $(d)$ \\
\hline PZT-8 & $250 \mu \mathrm{m}$ & $500 \mu \mathrm{m}$ & $250 \mu \mathrm{m}$ & $5 \mu \mathrm{m}$ \\
\hline
\end{tabular}


To satisfy the constraint of dielectric displacement $D_{i, j}$ expressed in (1i), the differential of $D_{z}$ is required to be zero. Considering Kirchhoff's theory $(D r=0)$, we get a constraint from $(7 \mathrm{c})$,

$$
\frac{\partial D_{z}}{\partial z}=e_{31}^{p}\left(\frac{\partial u_{r}^{2}}{\partial r \partial z}+\frac{1}{r} \frac{\partial u_{r}}{\partial z}\right)=0
$$

using the conventional assumption with decoupled $u_{r}$ and $u_{z}$. Eq. (9) is easy to satisfy because the component $\left(\partial u_{r}^{2} / \partial r \partial z+(1 / r)\left(\partial u_{r} / \partial z\right)\right)$ is obviously zero, but this results in disagreement between model and experiment results. However, in our new assumption, only suitable values of $u_{r}$ and $u_{z}$ are able to make that component zero. A potential solution is apparent if $e_{31}^{p}$ is set to be zero. Eq. (9) is satisfied, but it leads to no piezoelectricity of the material. To avoid this problem, we can make another approximation for radial displacement $u_{r}$ to satisfy (9):

$$
u_{r}=\left(\frac{z^{2}}{2}+C\right) \cdot \frac{\partial w}{\partial r} \approx C \cdot \frac{\partial w}{\partial r} .
$$

It is assumed that $\left(Z^{2} / 2\right)$ is very small compared with the unknown constant $C$. The validity of this approximation will be demonstrated later. Therefore, the radial displacement $u_{r}$ is only a function of the variable radius $r$, and thus $\partial D z / \partial z=0$ is satisfied. Using (2)-(8) and (10), we can then get

$$
\frac{\partial u_{r}^{2}}{\partial r^{2}}+\frac{1}{r} \frac{\partial u_{r}}{\partial r}+\left(k^{2}-\frac{1}{r^{2}}\right) u_{r}=0
$$

where $k^{2}=\rho \omega^{2} / c_{11}{ }^{p}$ is the wave number. The general solution of (11) is

$$
u_{r}=A J_{1}(k r)+B Y_{1}(k r),
$$

where $J_{1}$ is the Bessel function of the first kind and first order, $Y_{1}$ is the Bessel function of the second kind and first order. $A$ and $B$ are constants determined by the boundary conditions. The stress-free boundary condition is that $T_{r}=0$ at the inner and outer surface (at $\left.r=a, b\right)$. By using the boundary condition, we obtain

$$
\left|\begin{array}{ll}
a_{11} & a_{12} \\
a_{21} & a_{22}
\end{array}\right| \cdot\left|\begin{array}{l}
A \\
B
\end{array}\right|=\left|\begin{array}{l}
b_{1} \\
b_{2}
\end{array}\right| \cdot V
$$

where $V$ is applied voltage, and other constants except $A$ and $B$ are expressed as

$$
\begin{gathered}
a_{11}=c_{11}^{p} k \cdot J_{0}(k a)+\left(c_{12}^{p}-c_{11}^{p}\right) \cdot J_{1}(k a) / a, \\
a_{12}=c_{11}^{p} k \cdot Y_{0}(k a)+\left(c_{12}^{p}-c_{11}^{p}\right) \cdot Y_{1}(k a) / a, \\
a_{21}=c_{11}^{p} k \cdot J_{0}(k b)+\left(c_{12}^{p}-c_{11}^{p}\right) \cdot J_{1}(k b) / b, \\
a_{22}=c_{11}^{p} k \cdot Y_{0}(k b)+\left(c_{12}^{p}-c_{11}^{p}\right) \cdot Y_{1}(k b) / b, \\
b_{1}=b_{2}=-e_{31}^{p} / d .
\end{gathered}
$$

From (13) and (14), constants $A$ and $B$ can be determined,

$$
A=A_{0} V, \quad B=B_{0} V,
$$

where the value of constants $A_{0}$ and $B_{0}$ is calculated from the following equation:

$$
\left|\begin{array}{l}
A_{0} \\
B_{0}
\end{array}\right|=\left|\begin{array}{ll}
a_{11} & a_{12} \\
a_{21} & a_{22}
\end{array}\right|^{-1} \cdot\left|\begin{array}{l}
b_{1} \\
b_{2}
\end{array}\right| .
$$

To verify the approximation used in (10), the unknown constant $C$ needs to be defined from the zero thickness stress $\left(T_{z}=0\right)$ assumption in Kirchhoff's theory:

$$
T_{z}=c_{13}^{E}\left(\frac{\partial u_{r}}{\partial r}+\frac{u_{r}}{r}\right)+\frac{c_{33}^{E} \partial u_{z}}{\partial r}+e_{33} \frac{\partial \varphi}{\partial z}=0 .
$$

From (10) and (12), w(r) can be deduced,

$$
\begin{aligned}
\partial w(r) / \partial r & =\left[A J_{1}(k r)+B Y_{1}(k r)\right] / C, \\
w(r) & =-\left[A J_{0}(k r)+B Y_{0}(k r)\right] / k C+D,
\end{aligned}
$$

where $D$ is another unknown constant from integration. Substituting $u_{r}$ and $u_{z}$ into (17),

$$
\left(c_{13}^{E} k+\frac{c_{33}^{E}}{k C}\right) \cdot\left[A J_{1}(k r)+B Y_{1}(k r)\right]+\frac{e_{33} V}{d}-c_{33}^{E} D=0 .
$$

To make (19) valid, the components $\left(c_{13}^{E} k+c_{33}^{E} / k C\right)$ and $\left(e_{33} V / d-c{ }_{33}^{E} D\right)$ need to be zero, thus, the constants $C$ and $D$ are obtained:

$$
C=-\frac{c_{33}^{E}}{c_{13}^{E} k^{2}}, \quad D=\frac{e_{33}}{c_{33}^{E} d} V .
$$

It can be seen that $C$ is a frequency-dependent constant. Remembering that, in the approximation made in (10), $\left(Z^{2} / 2\right)$ is assumed to be a small value that can be ignored. It is essential to check if it is valid. Fig. 3 illustrates the influence of function $Y=\left(Z^{2} / 2\right)+C$ compared with function $Y=C$, demonstrating that the component $\left(Z^{2} / 2\right)$ is sufficiently small to be ignored.

Therefore, $u_{r}$ and $u_{z}$, meeting the constraints and Kirchhoff's theory, are successfully deduced as

$$
\begin{aligned}
& u_{z}=-z \cdot\left\{\frac{c_{13}^{E}}{c_{33}^{E}} k\left[A J_{0}(k r)+B Y_{0}(k r)\right]+\frac{e_{33}}{c_{33}^{E} d}\right\} \cdot V, \\
& u_{r}=\left[A_{0} J_{1}(k r)+B_{0} Y_{1}(k r)\right] \cdot V .
\end{aligned}
$$

As far as the electric part is concerned, by using the equation below,

$$
I=j \omega Q=-j \omega 2 \pi \int_{a}^{b} r D_{z} \mathrm{~d} r
$$




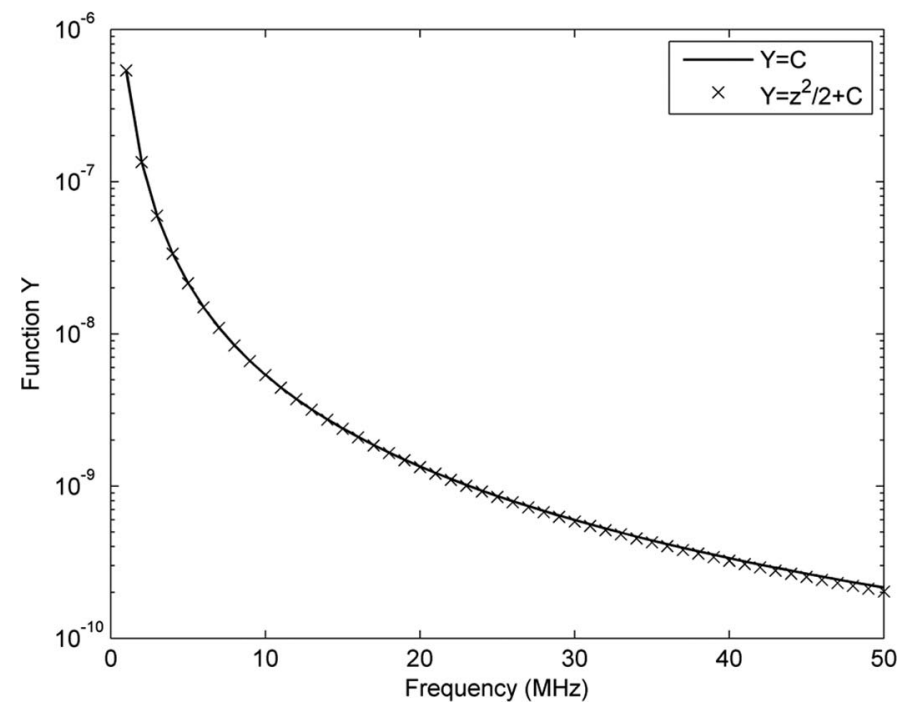

Fig. 3. Comparison of $C$ and $\left(Z^{2} / 2+C\right)$ at thickness $Z= \pm d / 2$.

the admittance $Y$ and impedance $\mathrm{Z}$ can be obtained,

$$
Y=j \omega C_{0}+Y_{1}+Y_{2}, \quad Z=1 / Y,
$$

where the parameters are shown as follows. $C_{0}$ is clamped capacitance and $S$ is surface area of the ring:

$$
\begin{aligned}
& C_{0}=\varepsilon_{33}^{p} S / d, \quad S=\pi\left(b^{2}-a^{2}\right), \\
& Y_{1}=-j 2 \pi \omega \cdot e_{31}^{p}\left[b J_{1}(k b)+a J_{1}(k a)\right] \cdot A_{0}, \\
& Y_{2}=-j 2 \pi \omega \cdot e_{31}^{p}\left[b Y_{1}(k b)+a Y_{1}(k a)\right] \cdot B_{0} .
\end{aligned}
$$

\section{Theoretical And FEA Results}

Calculation of the resonant frequency of a ring is not trivial. We can get an estimate by using the rule obtained by Lula et al. [1], $f_{m}<f_{0}<f_{d}$, where $f_{0}$ is the fundamental resonant frequency of ring, $f_{d}$ is the resonances of a disk membrane with the same outer radius as the ring, and $f_{m}$ is the resonance of a thin ring whose inner radius approaches its outer radius (i.e., a very small width); $f_{m}$ can be obtained by the Berlincourt model [11]. If the radius of a ring membrane needs to be about $500 \mu \mathrm{m}$, then the fundamental frequency $f_{m}$ for the example ring can be calculated as $1 \mathrm{MHz}$, while for a disk through the theory described in [9], the fundamental frequency $f_{d}$ is found to be around $2 \mathrm{MHz}$. According to (24), $f_{0}$ ranges from 1 to

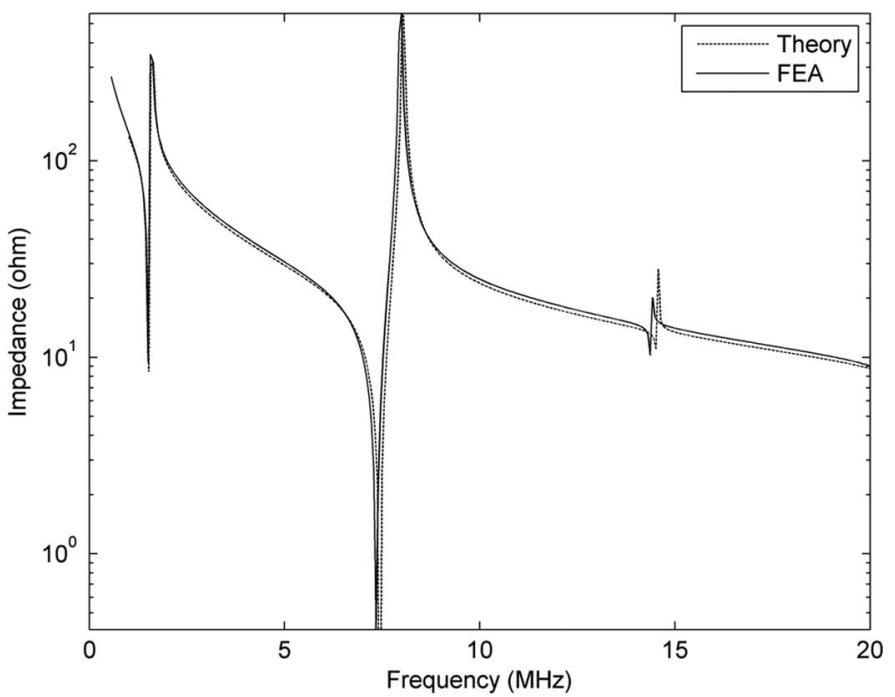

Fig. 4. The impedance from analytical theory and finite element analysis (FEA).

$2 \mathrm{MHz}$. Thus, for the ring as designed in Table I we would expect a fundamental resonance of about $1.5 \mathrm{MHz}$.

Fig. 4 shows the theoretical results of impedance from our model, compared with the FEA results from ANSYS. The correlation is very good, and it can be seen that the fundamental resonance is indeed at about $1.5 \mathrm{MHz}$. Table II lists the value of the resonant and antiresonant peaks from calculation and FEA simulation. Higher R modes also show good agreement, but are not listed here.

In the following figures, simulation results by FEA and computation results by the theory developed in this paper are illustrated for both $u_{r}$ and $u_{z}$ in the radial direction. All the displacements are for an applied voltage source of $1 \mathrm{~V}$, and the figures show the results for the surface of half the ring, starting from a inner radius of $250 \mu \mathrm{m}$ and finishing at an outer radius of $500 \mu \mathrm{m}$. In Fig. 5, theory and FEA results of displacement at the 1 st $\mathrm{R}$ mode are given. Both of the figures show good agreement between the theory and FEA results. Likewise, Figs. 6 and 7 show these displacements for the 2nd and 3rd R mode, respectively.

Both the FEA and theory results are well matched; $u_{r}$ displacement at these $\mathrm{R}$ modes agrees with the typical thin ring vibrations analyzed by Iula [1]. More importantly, the expected displacement $u_{z}$ is shown well by the analytical model and shows a sinusoidal-shape bending vibration, especially for the high-order resonance. Fig. 7(b) illustrates the resonant mode having nodal circles, for example, at a radial position of $0.375 \mathrm{~mm}$, which

Table II. Values of Resonant Frequencies From Theoretical Model and Finite Element Analysis

(FEA).

\begin{tabular}{lcccc}
\hline Mode & $\begin{array}{c}\text { Resonance by } \\
\text { theory }(\mathrm{MHz})\end{array}$ & $\begin{array}{c}\text { Resonance by } \\
\text { FEA }(\mathrm{MHz})\end{array}$ & $\begin{array}{c}\text { Antiresonance by } \\
\text { theory }(\mathrm{MHz})\end{array}$ & $\begin{array}{c}\text { Antiresonance by } \\
\text { FEA (MHz) }\end{array}$ \\
\hline 1st mode & 1.495 & 1.475 & 1.609 & 1.570 \\
2nd mode & 7.445 & 7.365 & 8.044 & 7.983 \\
3rd mode & 14.50 & 14.35 & 14.58 & 14.40 \\
\hline
\end{tabular}




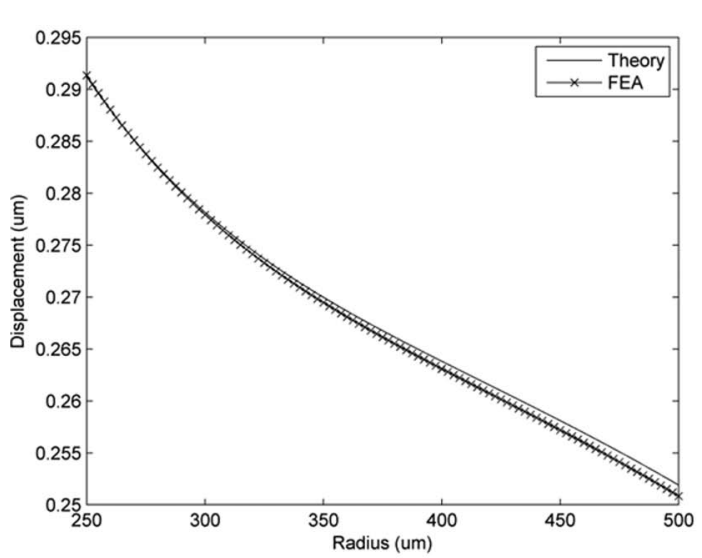

(a)

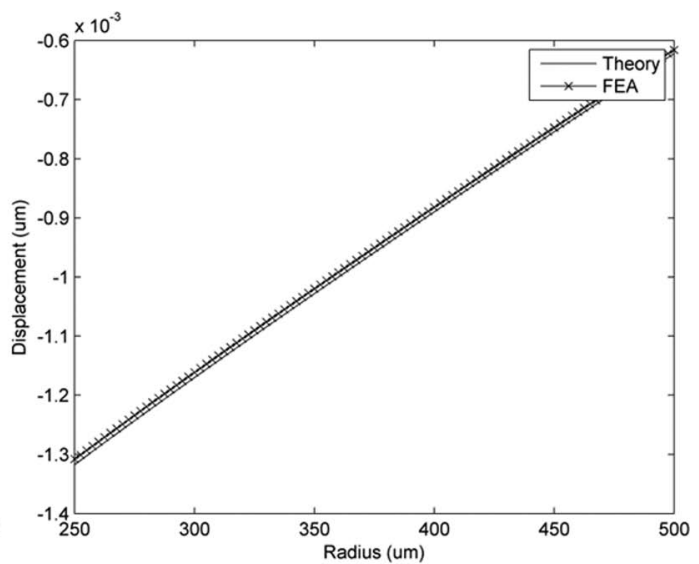

(b)

Fig. 5. Theory and inite element analysis (FEA) results of (a) $u_{r}$ and (b) $u_{z}$ along radial direction at 1 st $\mathrm{R}$ mode.

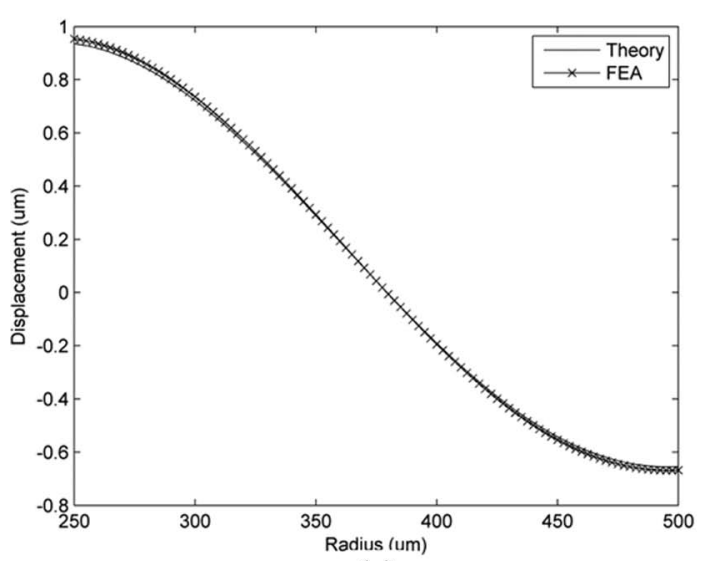

(a)

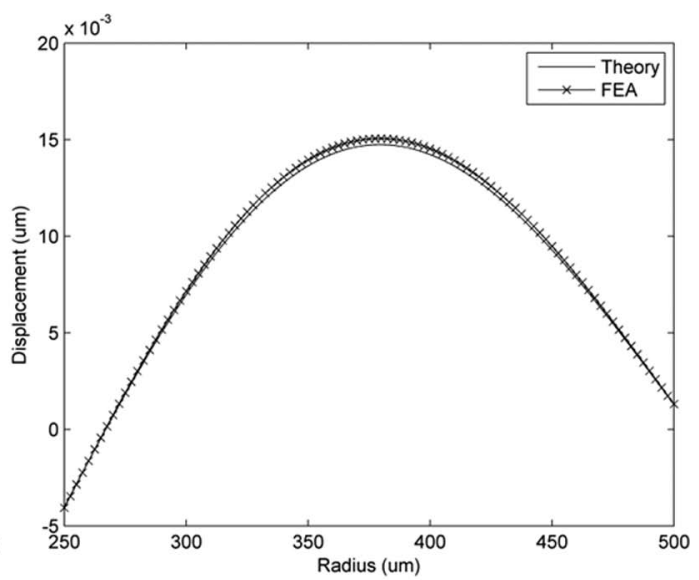

(b)

Fig. 6. Theory and inite element analysis (FEA) results of (a) $u_{r}$ and (b) $u_{z}$ along radial direction at $2 \mathrm{nd} \mathrm{R}$ mode.

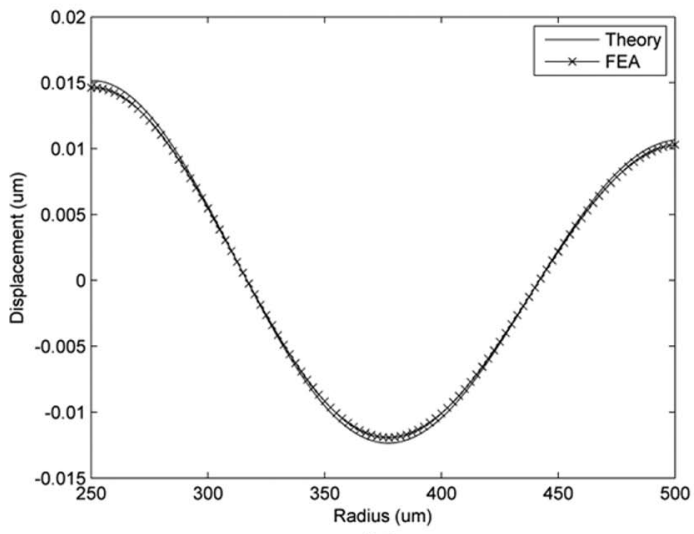

(a)

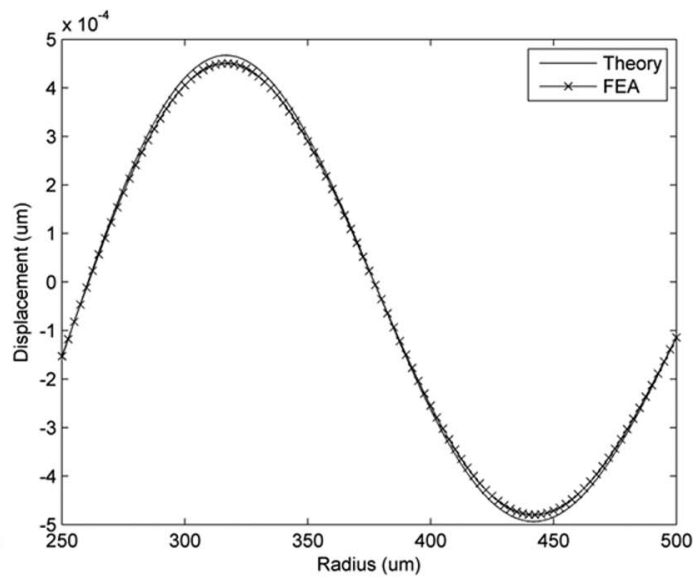

(b)

Fig. 7. Theory and inite element analysis (FEA) results of (a) $u_{r}$ and (b) $u_{z}$ along radial direction at 3rd $\mathrm{R}$ mode.

is generated by the coupled vibration due to $\mathrm{R}$ modes. More nodal circles can be found by increasing the order of resonance. At higher frequencies where the TE mode can be generated, the $u_{z}$ bending obtained from the coupling of higher order $\mathrm{R}$ modes in this model will couple into the TE vibration.

\section{CONCLUSION}

To conclude, a new 2-D model of a thin ring transducer has been presented, and both radial and thickness displacements are expressed and shown to match the results given by FEA. This validates the new modified assump- 
tion in our analytical model to realize a coupled $2-\mathrm{D}$ vibration, instead of the assumption of decoupled $u_{r}$ and $u_{z}$ used by previous work. This new approach shows excellent agreement with FEA solutions when considering radial modes. The proposed radial vibration is reasonable, and the bending is discovered and analyzed well by our model. At higher order modes in particular, it also shows nodal circles formed as a result of the coupling of $u_{r}$ and $u_{z}$ from $\mathrm{R}$ modes, implying that the sinusoidal-shape bending will have an impact on the TE mode in far-frequency range. This effect has been associated with high order $\mathrm{R}$ modes, but this paper shows a possible mechanism that adds credibility to this thesis. In addition, because the assumption here is based on coupled vibration, it is quite promising to extend this model into 3 dimensions and consider coupling between TE and $\mathrm{R}$ modes in far-frequency range to investigate this point further.

\section{REFERENCES}

[1] A. Iula, N. Lamberti, and M. Pappalardo, "A model for the theoretical characterization of thin piezoceramic rings," IEEE Trans. Ultrason. Ferroelectr. Freq. Control, vol. 43, no. 3, pp. 370-375, May 1996.

[2] H. Chi-Hung, M. Chien-Ching, and L. Yu-Chih, "Theoretical, numerical, and experimental investigation on resonant vibrations of piezoceramic annular disks," IEEE Trans. Ultrason. Ferroelectr. Freq. Control, vol. 52, no. 8, pp. 1204-1216, Aug. 2005.

[3] S. Y. Lin, "Electro-mechanical equivalent circuit of a piezoelectric ceramic thin circular ring in radial vibration," Sens. Actuators A Phys., vol. 134, no. 2, pp. 505-512, 2007.

[4] A. Iula, N. Lamberti, and M. Pappalardo, "An approximated 3-D model of cylinder-shaped piezoceramic elements for transducer design," IEEE Trans. Ultrason. Ferroelectr. Freq. Control, vol. 45, no. 4, pp. 1056-1064, Jul. 1998.

[5] F. Fei, J. Shen, and J. Deng, "A 2-D equivalent circuit of piezoelectric ceramic ring for transducer design," Ultrasonics, vol. 44, pp. e723-e726, Dec. 2006.

[6] S. Y. Lin, "Study on the equivalent circuit and coupled vibration for the longitudinally polarized piezoelectric ceramic hollow cylinders," J. Sound Vibrat., vol. 275, no. 3-5, pp. 859-875, 2004.

[7] N. Lamberti and M. Pappalardo, "A general approximated twodimensional model for piezoelectric array elements," IEEE Trans. Ultrason. Ferroelectr. Freq. Control, vol. 42, no. 2, pp. 243-252, Mar. 1995.

[8] L. Hing Leung, H. Jun Hui, and H. L. W. Chan, "Finite element analysis on piezoelectric ring transformer," IEEE Trans. Ultrason. Ferroelectr. Freq. Control, vol. 51, no. 10, pp. 1247-1254, Oct. 2004.

[9] A. H. Meitzler, H. M. O'Bryan Jr., and H. F. Tiersten, "Definition and measurement of radial mode coupling factors in piezoelectric ceramic materials with large variations in poisson's ratio," IEEE Trans. Sonics Ultrason., vol. 20, no. 3, pp. 233-239, 1973.

[10] G. Percin and B. T. Khuri-Yakub, "Piezoelectrically actuated flextensional micromachined ultrasound transducers. I. Theory," IEEE Trans. Ultrason. Ferroelectr. Freq. Control, vol. 49, no. 5, pp. 573$584,2002$.

[11] D. A. Berlincourt, D. R. Curran, and H. Jaffe, "Piezoelectric and piezomagnetic material and their function in transducers," in Physical Acoustics, vol. 1, W. P. Mason, Ed. New York: Academic Press, 1964.

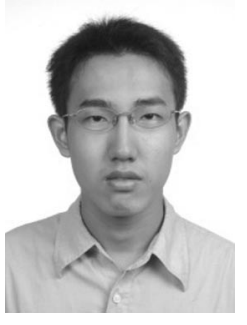

Yichen Qian was born in Shanghai, China, in 1985. He received the B.Sc. degree in electronic science and technology from the School of Material, University of Shanghai, Shanghai, China, in 2007. He is currently working toward a Ph.D. degree in the School of Electronics and Computer Science, University of Southampton, Southampton, UK. His research area is ultrasonic transducers, mainly for medical applications.

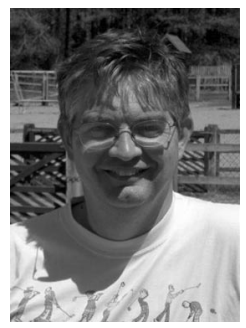

Nick Harris is a lecturer in the School of Electronics and Computer Science at the University of Southampton, Southampton, UK, and he has more than 75 publications in the fields of wireless sensor networks, biosensors, microfluidic systems, and processing. He was awarded a Ph.D. degree in 1997 by the University of Southampton, Department of Mechanical Engineering. In 1998, Dr. Harris was appointed principal engineer at ERA Technology in the Microsystems and Materials Group before returning to the University of Southampton as a senior research fellow with research interests in miniaturized fluidic systems resulting in a unique type of microfluidic particle concentrator using ultrasonic standing waves. Current research areas include self-powered health and usage monitors and embedded condition-monitoring microsystems.

His other research interests include wireless sensor networks and analogue and digital electronic sensor circuit design. He is a member of the IET and a Chartered Engineer, and a co-founder of Perpetuum Ltd, an energy-harvesting spin-off company from Southampton.

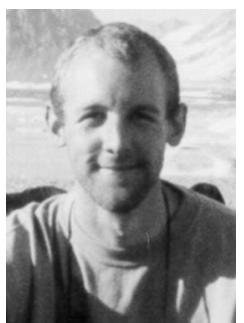

Peter Glynne-Jones graduated with first class honors from the School of Electronics and Computer Science at the University of Southampton, Southampton, UK. He was sponsored through his degree by the Defense Engineering and Science Group and was an IEE scholar. He was awarded a $\mathrm{Ph} . \mathrm{D}$. degree from the University of Southampton in 2001; his dissertation was entitled "Vibration powered generators for self-powered microsystems." The spin-off company Perpetuum was formed to commercialize designs developed as part of his Ph.D. work.

After his Ph.D. studies, Dr. Glynne-Jones spent four years living in a Buddhist monastery before returning to the University of Southampton as a research fellow, working on the manipulation of particles in microsystems using ultrasonic radiation forces.

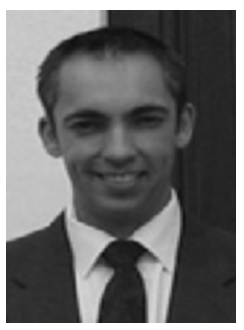

Steve Beeby obtained a $\mathrm{Ph} . \mathrm{D}$. degree from the University of Southampton, Southampton, UK, in 1998 on the subject of micromechanical resonators. He was awarded a prestigious EPSRC Advanced Research Fellowship in 2001 to investigate the combination of screen-printed piezoelectric materials with micromachined structures. He is currently a reader in the School of Electronics and Computer Science at the University of Southampton. His research interests include energy harvesting, MEMS, active printed materials development and biometrics. He is the coordinator of a European Union Framework Integrated Project called "MICROFLEX" and is principal or co-investigator on 3 additional projects. He is a co-founder of Perpetuum Ltd., a university spin-off company based on vibration energy harvesting formed in 2004. He has co-authored one book, MEMS Mechanical Sensors, published more than 125 publications in the field, and holds 5 patents. 